The Astrophysical Journal, 613:886-897, 2004 October 1

(C) 2004. The American Astronomical Society. All rights reserved. Printed in U.S.A.

\title{
THE STELLAR DISK THICKNESS OF LOW SURFACE BRIGHTNESS GALAXIES
}

\author{
D. BIZYAEV ${ }^{1,2,3}$ and S. KaJsin ${ }^{4}$ \\ Received 2003 October 23; accepted 2004 June 9
}

\begin{abstract}
We present surface photometry results for a sample of 11 edge-on galaxies observed with the $6 \mathrm{~m}$ telescope at the Special Astrophysical Observatory, Russia. The photometric scale length, scale height, and central surface brightness of the stellar disks of our sample galaxies are estimated. We show that four galaxies in our sample, which are visually classified as objects of the lowest surface brightness class in the Revised Flat Galaxy Catalog, have bona fide low surface brightness (LSB) disks. We find from a comparison of photometric scales that the stellar disks of LSB galaxies are thinner than those of high surface brightness (HSB) galaxies. There is a clear correlation between the central surface brightness of the stellar disk and its vertical-to-radial scale ratio. The masses of spherical subsystems (dark halo + bulge) and the dark halo masses are obtained for the sample galaxies based on the thickness of their stellar disks. The LSB galaxies tend to harbor more massive spherical subsystems than the HSB objects, whereas no systematic difference in the dark halo masses is found between LSB and HSB galaxies. At the same time, the inferred mass-to-luminosity ratio for the LSB disks appears to be systematically higher than for HSB disks.
\end{abstract}

Subject headings: dark matter — galaxies: spiral — galaxies: structure

\section{INTRODUCTION}

Low surface brightness (LSB) spiral galaxies have been studied extensively in recent years. The main feature that distinguishes them from "regular," high surface brightness (HSB) galaxies is the roughly 2 mag lower central surface brightness of their stellar disks. They are thought to harbor massive dark halos (de Blok et al. 2003). The LSB rotation curves are shallower in their central parts (McGaugh et al. 2001), which points to a large dark matter fraction.

By observing the thickness of the stellar disk in a galaxy, one can constrain the relative mass of the dark halo (Zasov et al. 1991). Until recently, however, only a few edge-on LSB galaxies have been explored in detail (e.g., UGC 7321, Matthews 2000; IC 5249, van der Kruit et al. 2001).

We conducted a study of a small uniform sample of LSB and HSB galaxies observed with the same instrument to compare their structural parameters. Here we present the results of photometric observations in the $V$ and $R$ bands of a sample of 11 edge-on galaxies. The paper is structured as follows. In $\S 2$ we describe the sample of galaxies and observations, in $\S 3$ we discuss the data reduction and present the structural parameters of our galaxies, in $\S 4$ we use the inferred disk thickness to estimate the dark halo mass, $\S 5$ contains a discussion of selection effects and relations between the inferred parameters, and the main results are summarized in $\S 6$.

\section{SAMPLE OF GALAXIES AND OBSERVATIONS}

Our sample is based on the Revised Flat Galaxy Catalog (RFGC; Karachentsev et al. 1999). All galaxies included in this catalog are highly inclined objects. We selected objects from

\footnotetext{
${ }^{1}$ Physics Department, University of Texas at El Paso, 500 West University Avenue, El Paso, TX 79968.

2 Sternberg Astronomical Institute, University of Moscow, Moscow 119899, Russia; dmbiz@sai.msu.ru.

${ }^{3}$ Isaac Newton Institute of Chile, Moscow Branch, Russia.

4 Special Astrophysical Observatory of the Russian Academy of Sciences, pos. Nizhnij Arkhyz 357147, Karachaevo-Cherkessia, Russia; skai@sao.ru.
}

the faintest surface brightness (SB) class (IV, according to the RFGC) as candidates for LSB galaxies, and objects from intermediate or high surface brightness classes as reference HSB objects. We narrowed the sample of objects to galaxies large enough for structural studies (major axis size $>2^{\prime}$ in the RFGC) that fit inside the 3.' 5 field of view of our CCD imager. In three observing nights of our program we obtained data for 11 galaxies.

Photometric observations were performed with the primefocus camera on the $6 \mathrm{~m}$ telescope at the Special Astrophysical Observatory of the Russian Academy of Sciences. This setup provides a plate scale of 0.2 pixel $^{-1}$ and good sensitivity for faint regions of galaxies. The data were taken on 2000 April 27, 28 , and 30 in the Johnson-Cousins $V$ and $R$ photometric bands. The $V$-band images were used mostly for calibration purposes, while the $R$-band images were used for the measurements of structural parameters. For most of the galaxies in our sample we made two to four images shifted by a few pixels in both photometric bands.

The Landolt photometric standards (Landolt 1992) were observed every night. Table 1 summarizes our observations, listing object names, SB class (according to the RFGC), date of the observation, total integration time in every photometric band, number of exposures, and average seeing when the target was observed.

\section{DATA REDUCTION AND RESULTS}

The data were reduced using standard tools in the MIDAS package. The images of galaxies and photometric standards were corrected for the bias and dark current and flat-fielded. The images were then sky subtracted, aligned, and combined. We checked the quality of flat-fielding and sky subtraction by comparing the background level in those parts of the image that are free of foreground stars and are located close to a sample galaxy. The large-scale pattern of the background does not introduce uncertainties above $0.1 \%$. Three galaxies have very bright stars in their fields, which raises the large-scale background fluctuations to $0.2 \%$. 
TABLE 1

Summary of the ObServing Run

\begin{tabular}{|c|c|c|c|c|c|c|c|c|}
\hline Name & SB Class & Band & $\begin{array}{c}\text { Date } \\
(1999)\end{array}$ & $\begin{array}{c}\text { Integration Time } \\
\text { (s) }\end{array}$ & Exposure & $\begin{array}{l}\text { Seeing } \\
(\operatorname{arcsec})\end{array}$ & $\begin{array}{c}\text { Sky } \\
\left(\mathrm{mag} \operatorname{arcsec}^{-2}\right)\end{array}$ & $\begin{array}{l}\mathrm{S} / \mathrm{N}=3 \text { Level } \\
\left(\mathrm{mag} \operatorname{arcsec}^{-2}\right)\end{array}$ \\
\hline \multirow[t]{2}{*}{ UGC $10111 \ldots \ldots \ldots}$. & \multirow[t]{2}{*}{ IV } & $V$ & Apr 27 & 600 & 1 & 1.9 & 21.37 & 26.58 \\
\hline & & $R$ & Apr 27 & 1200 & 4 & 2.0 & 20.72 & 26.77 \\
\hline \multirow[t]{2}{*}{ UGC $11301 \ldots \ldots \ldots}$. & \multirow[t]{2}{*}{ III } & $V$ & Apr 27 & 700 & 3 & 1.6 & 20.76 & 25.17 \\
\hline & & $R$ & Apr 27 & 1000 & 4 & 1.6 & 20.38 & 25.81 \\
\hline \multirow[t]{2}{*}{ UGC $5662 \ldots \ldots \ldots \ldots$} & \multirow[t]{2}{*}{ III } & $V$ & Apr 30 & 600 & 1 & 3.0 & 21.39 & 26.64 \\
\hline & & $R$ & Apr 30 & 1200 & 2 & 2.4 & 20.59 & 26.63 \\
\hline \multirow[t]{2}{*}{ UGC $6080 \ldots \ldots \ldots \ldots . . .}$. & \multirow[t]{2}{*}{ II } & $V$ & Apr 30 & 600 & 1 & 1.9 & 21.49 & 26.46 \\
\hline & & $R$ & Apr 30 & 1200 & 2 & 1.7 & 20.68 & 26.60 \\
\hline \multirow[t]{2}{*}{ UGC $6686 \ldots \ldots \ldots \ldots$} & \multirow[t]{2}{*}{ III } & $V$ & Apr 27 & 1200 & 2 & 1.8 & 21.35 & 26.85 \\
\hline & & $R$ & Apr 27 & 900 & 3 & 1.7 & 20.48 & 26.45 \\
\hline \multirow[t]{2}{*}{ UGC $7808 \ldots \ldots \ldots \ldots . . .}$. & \multirow[t]{2}{*}{ IV } & $V$ & Apr 27 & 600 & 1 & 2.2 & 21.35 & 26.40 \\
\hline & & $R$ & Apr 27 & 1200 & 2 & 2.0 & 20.56 & 26.66 \\
\hline \multirow[t]{2}{*}{ UGC $9138 \ldots \ldots \ldots \ldots . .}$. & \multirow[t]{2}{*}{ I } & $V$ & Apr 28 & 900 & 2 & 1.1 & 21.37 & 26.48 \\
\hline & & $R$ & Apr 28 & 900 & 2 & 1.0 & 20.63 & 26.90 \\
\hline UGC $9422 \ldots \ldots \ldots \ldots . .$. & I & $R$ & Apr 30 & 1200 & 2 & 1.7 & 20.57 & 26.91 \\
\hline \multirow[t]{2}{*}{ UGC $9556 \ldots \ldots \ldots \ldots . . . .}$. & \multirow[t]{2}{*}{ IV } & $V$ & Apr 28 & 1800 & 4 & 1.0 & 21.46 & 26.51 \\
\hline & & $R$ & Apr 28 & 2900 & 6 & 1.0 & 20.68 & 27.26 \\
\hline \multirow[t]{2}{*}{ FGC $1273 \ldots \ldots \ldots . .}$. & \multirow[t]{2}{*}{ IV } & $V$ & Apr 27 & 600 & 2 & 1.8 & 21.43 & 26.54 \\
\hline & & $R$ & Apr 27 & 900 & 2 & 1.7 & 20.50 & 26.59 \\
\hline NGC $4738 \ldots \ldots \ldots \ldots . . . .$. & $\mathrm{I}$ & $R$ & Apr 30 & 1200 & 2 & 1.5 & 20.45 & 26.56 \\
\hline
\end{tabular}

NoтE.-Name of galaxy, SB class (according to the RFGC catalog), photometric band, date of observation, exposure time (total), number of exposures, average seeing, level of sky brightness, and $\mathrm{S} / \mathrm{N}=3 \mathrm{SB}$ level in combined frames.

Eight and 12 Landolt's stars from three selected areas were available on April 27 and 30, respectively. The residuals for photometric solutions were $0.02 \mathrm{mag}$ for April 27 (in both $V$ and $R$ bands) and 0.04 mag (in both $V$ and $R$ bands) for the night of April 30. The sky brightness level is tabulated in Table 1. The surface brightness corresponding to a $3 \sigma$ level of the background noise in the final combined images is shown in Table 1 as well.

The observing conditions were nonphotometric during part of the night on April 28. However, most of our galaxies have the major axis photometric profiles in the $R$ band published by Karachentsev et al. (1992). This enables us to verify the calibration and to adjust it for the nonphotometric night. The mean difference between the surface brightnesses we derived and those published by Karachentsev et al. (1992) is of the order of $0.3 \mathrm{mag}$. The largest source of discrepancy is the use of different procedures of the major axis profiles extraction.

Comparison of the sky brightness in $R$ images can be used to roughly estimate the zero point of calibration for the objects observed on April 28. If we use this method of calibration, the $R$-band surface brightnesses of UGC 9138 and UGC 9556 would be $0.4 \mathrm{mag}$ lower than those used in this paper.

The combined and calibrated images were used to obtain the radial scale length $h$, vertical scale height $z_{0}$, and "face-on" central surface brightness of the stellar disk, as well as the bulge-to-disk luminosity ratio $L_{b} / L_{d}$.

The images were rotated to align the galactic plane parallel to the horizontal axis. Choosing the rotation angle, we direct our attention to the intermediate regions of galactic disks, where a possible bulge does not reveal itself and the signal-tonoise ratio $(\mathrm{S} / \mathrm{N})$ is high enough.

We applied a standard method (van der Kruit \& Searle 1981) to derive the structural parameters extracting photometric profiles parallel to the major and minor galactic axes. The radial scale length was obtained from two photometric profiles extracted parallel to the major axis and displaced with respect to the galactic midplane. This allows us to minimize the effects of dust absorption, because we avoid the galactic midplane. An average displacement is of the order of $0.7 z_{0}$ (see below). If the bulge was present, the central part of the radial photometric profiles (typically, $1 \mathrm{~h}$ from the center) is excluded from further analysis. We fit the function $f(r)=2 I_{0} \operatorname{sech}^{2}\left(z / z_{0}\right)$ $\int_{0}^{R_{\max }} \exp (-l / h) r d l$ to the radial profiles and find mean values of $I_{0}$ and $h$. Here, $r$ is the distance to the center, and $R_{\max }$ is the distance to the edge of the disk. One can assume that $R_{\max }=4 h$ (Pohlen et al. 2002; Holley-Bockelmann \& Mihos 2001). The integration was made along the line of sight $l$. Two radial profiles used for the fitting are shown in the middle panels of Figures $1-11$ by the solid lines. Note that each profile was manually cleaned of foreground stars before the fitting. The radial profile drawn through the galactic plane is shown in the middle panels of Figures $1-11$ by the dashed line.

As a next step, we draw 10-14 cuts made parallel to the minor axis of a galaxy and fit each photometric profile with the function $f(z)=I \operatorname{sech}^{2}\left(|z+d z| / z_{0}\right)$. Here $|z|$ designates the distance to the galactic plane. The "displacement term" $d z$ enables us to correct the values of the disk scale height for a possible disorientation of the major axis or disk warp. The resulting value of the scale height $z_{0}$ and its error were found by averaging the values throughout the disk. Our galaxies show no significant variations of scale height with radius. Hence, we defined the mean scale height with no weights.

Fitting the profiles, we convolved the function $f(z)$ with the Gaussian smearing function assuming its FWHM from Table 1. The corresponding vertical profiles are shown in the top frames of Figures 1-11. They were manually cleaned of the foreground stars and artifacts before the fitting.

The value of the disk central surface brightness $\mu_{0}$ corrected to the face-on inclination was calculated with the parameters $I_{0}$ and $z_{0}$ inferred above, taking into account the photometric 

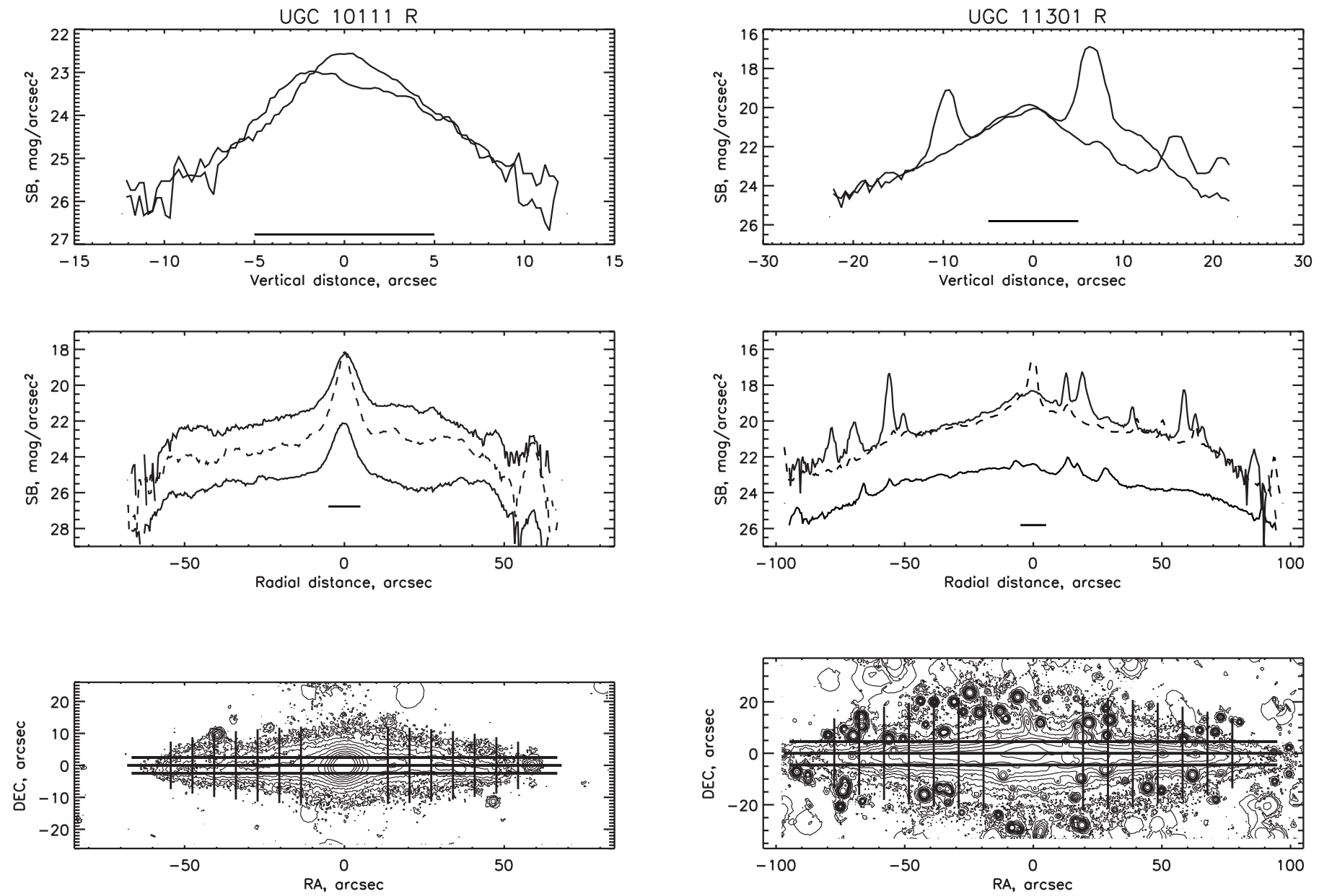

FIG. 1.-Top: Examples of the vertical profiles of UGC 10111 extracted parallel to its minor axis. Both lines show profiles taken along two vertical cuts closest to the galactic center (see isophotal map in the bottom panel). The $10^{\prime \prime}$ bar indicates the level of $\mathrm{S} / \mathrm{N}=3$. Middle: Radial profiles extracted along the major axis (dashed line) and parallel to it (solid lines). The latter were used to derive the structural parameters of the galaxy. Upper and lower lines are shifted from their real positions by +2 and -2 mag $\operatorname{arcsec}^{-2}$, respectively. They are extracted along the upper and lower radial cuts shown on the isophotal map. The $10^{\prime \prime}$ bar indicates the level of $\mathrm{S} / \mathrm{N}=3$. Bottom: Isophotal map of UGC 10111. The isophotes correspond to $20.5,21,21.5,22,22.5,23,23.5,24,24.5,25$, and $25.5 \mathrm{mag} \mathrm{arcsec}-2$ in the $R$ band. The places where the profiles were extracted are shown by lines. All artifacts and stars that could be seen in the picture were cleaned out manually before the structural parameters were found.

calibration equations. The extinction of our Galaxy (according to the LEDA database) is also included in the analysis and listed in Table 2.

In order to check how examining only a limited number of brightness profiles (two radial and 10-14 vertical) affects the inferred values, we derive the same values for each galaxy by extracting the radial profiles (drawn along the major axis) with an increment of 1 pixel from $0.2 z_{\max }$ to $0.8 z_{\max }$ in the vertical direction, where $z_{\max }$ is the minor axis of an ellipse encompassing the galaxy to the level of $\mathrm{S} / \mathrm{N}=3$. The vertical profiles in this analysis were drawn with 1 pixel increments taking a step off the disk edge and its center. The resulting structural parameters are similar to those obtained above using only a few photometric profiles. All conclusions of the paper remain unchanged in this case.

As was shown by de Grijs et al. (1997), we can neglect inclination corrections for inclinations larger than $86^{\circ}-87^{\circ}$. Our $V$ images are deep enough to see obscuration by dust in most of

FIG. 2.-Same as Fig. 1, but for UGC 11301. The isophotes correspond to $19.5,20.5,21,21.5,22,22.5,23,23.5$, and $24.5 \mathrm{mag} \mathrm{arcsec}^{-2}$.

our galaxies. Although dust is not seen in the galaxy FGC 1273, its bulge has a high degree of symmetry. Because its edge-on disk is very thin, we assume that its inclination angle is $90^{\circ}$. For all other galaxies we can estimate the inclination angle of the galactic plane from the shape and position of their dust layers and the asymmetric position of their centers of brightness with respect to external isophotes. The value of the inclination is shown in Table 3; its typical error is $0^{\circ} .7$. Based on those values, we applied no additional correction for non-edge-on inclination to the photometric parameters derived above.

Assuming the inferred disk parameters, we subtracted the disk and extracted the bulge images from the central parts of our galaxies. Then the central parts of two radial profiles mentioned above, as well as the vertical profiles extracted along the minor axis, were used to estimate the bulge parameters. The King profile, $\rho_{L}^{0}\left[1+\left(r / a_{b}^{k}\right)^{2}\right]^{-3 / 2}$, as well as the exponential one, $\rho_{L}^{0} \exp \left(-r / a_{b}^{e}\right)$, were used to fit the bulge volume luminosity density distribution. Here $\rho_{L}^{0}$ denotes the central volume luminosity density. The bulge scales $a_{b}^{k}$ and $a_{b}^{e}$ could be different in the vertical and radial directions (i.e., for oblate bulges). The inner parts of the vertical profiles were excluded from the analysis.

Bulges of most galaxies in our sample are best fitted by the King profile. The only exception is UGC 9556, the bulge of which is best fitted by the exponential profile. Because the central part of the latter galaxy is oblate, we suggest that it probably has two disks: an HSB disk encompassed by a more 

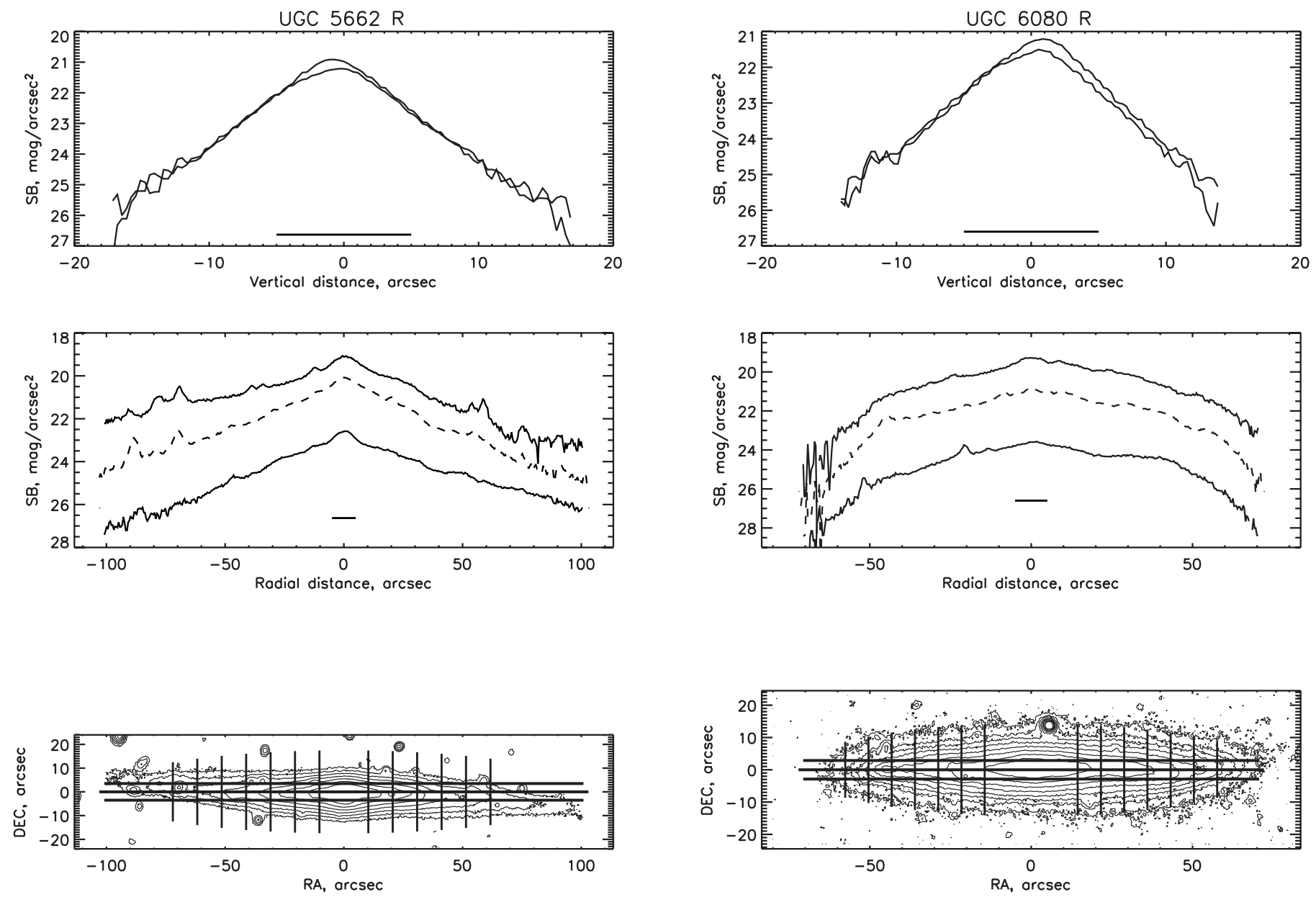

FIG. 3.- Same as Fig. 1, but for UGC 5662. The isophotes correspond to $20.5,21,21.5,22,22.5,23,23.5$, and $24 \mathrm{mag} \mathrm{arcsec}^{-2}$.

extended LSB disk. We consider its LSB disk throughout the paper. According to the RFGC, UGC 9556 may have a lens in its central part. On the other hand, its type was defined as a galaxy with a bar (SB?c) in the UGC catalog (Nilson 1973). Moreover, UGC 9556 has an asymmetry of bright isophotes close to the galactic plane, which points toward a possible bar shielded by dust whose nearer side is seen. Indeed, photometric identification of bars in edge-on galaxies can rarely be conclusive.

With the help of the parameters obtained, we infer the bulgeto-disk luminosity ratio $L_{b} / L_{d}$. The main results of the fitting are shown in Table 3 . The values of $h$ and $z_{0}$ are converted to the spatial units according to the adopted distances to the galaxies $D$. Table 3 also shows $R$ magnitudes and colors $(V-R)$ derived for our objects. The magnitudes were obtained by integrating background-subtracted images of the galaxies within elliptical diaphragms. Major and minor axes of the diaphragms correspond to the sizes from the RFGC, which are quite similar to the galaxies' dimensions at $\mathrm{S} / \mathrm{N}=3$ level.

The distribution of $\mu_{0}$ (see Table 3 ) indicates the presence of two subsamples: galaxies with $\mu_{0}$ greater than $23.5 \mathrm{mag}$ $\operatorname{arcsec}^{-2}$, which we define as LSB galaxies, and galaxies with a higher surface brightness, which are designated as HSB galaxies in this paper. Hence, our sample consists of four LSB and seven HSB galaxies. Note that all galaxies in the faintest RFGC SB class were classified here as LSB objects.

Although our sample of objects enables us to compare the structural parameters of LSB and HSB disks, the sample is very limited. We incorporated one more sample of edge-on

FIG. 4.-Same as Fig. 1, but for UGC 6080. The isophotes correspond to $20.5,21,21.5,22,22.5,23,23.5,24,24.5$, and $25.5 \mathrm{mag} \mathrm{arcsec}^{-2}$.

galaxies whose photometric parameters have been published by Barteldrees \& Dettmar (1994). They made use of similar red photometric band and technique to extract the photometric parameters. We utilize their data together with ours throughout the paper in order to increase the available sample of HSB galaxies. As it will be seen, the sample of Barteldrees \& Dettmar also includes one object that can be classified as an LSB galaxy.

\section{LSB VERSUS HSB: THE VERTICAL SCALE HEIGHT OF THE GALACTIC DISK AS A NEW FEATURE TO COMPARE}

As was shown in Bizyaev (2000), Bizyaev \& Mitronova (2002), and Reshetnikov et al. (2003), the galaxies of lower surface brightness tend to have smaller $z_{0} / h$ ratios. However, this conclusion was based on studies of mostly HSB galaxies. Now, we can incorporate our LSB subsample and consider the relation between $z_{0} / h$ and the central surface brightness $\mu_{0}$. The objects from our sample are denoted by squares in Figure 12. The open squares show the HSB subsample, whereas the filled ones designate LSB galaxies. The galaxies taken from Barteldrees \& Dettmar (1994) are shown by crosses.

Furthermore, the near-infrared $K_{s}$-band sample of edgeon galaxies from Bizyaev \& Mitronova (2002) (hereafter referred to as the 2MASS sample) is available for comparison. Here we must take into account the systematic difference in the brightness and $z_{0} / h$ between the $R$ and $K$ photometric bands. 

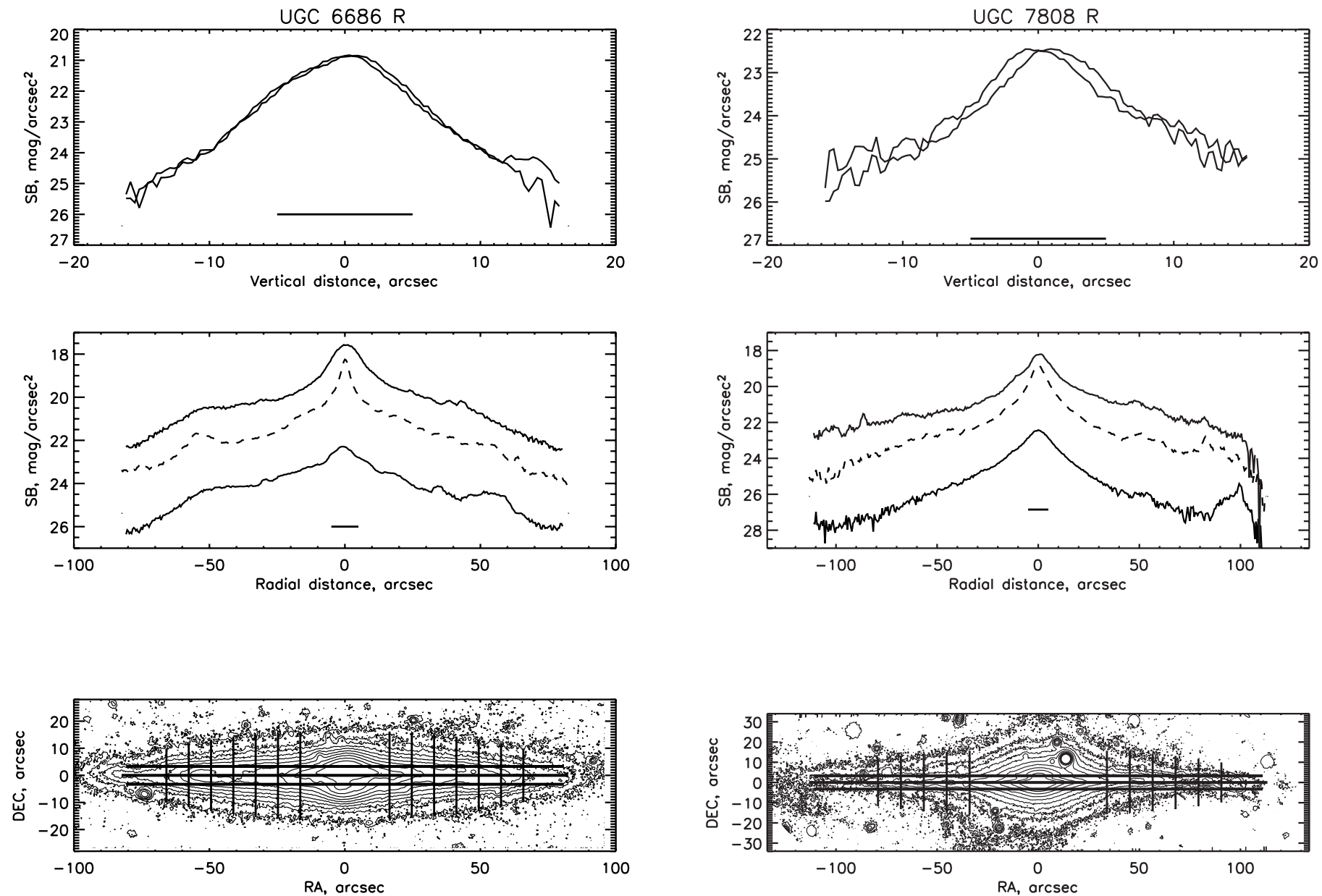

FIG. 5.-Same as Fig. 1, but for UGC 6686. The isophotes correspond to $20.5,21,21.5,22,22.5,23,23.5,24,24.5$, and $25.5 \mathrm{mag} \operatorname{arcsec}^{-2}$.

As noticed by Zasov et al. (2002), the ratio of scales $z_{0} / h$ is 1.4 times less for the stellar disk in $K$ than for the stellar disk in $R$. This can be explained by stronger dust extinction in the $R$ band and was well illustrated by Xilouris et al. (1998). We corrected $z_{0} / h$ for the 2MASS galaxies taken from Bizyaev \& Mitronova (2002) according to this value. The typical color $R-K=2.1 \mathrm{mag}$ inferred for late-type face-on spirals by de Jong (1996) was added to the central surface brightnesses of the 2MASS galaxies as well. The final correction we applied was the addition of the internal extinction to the 2MASS central surface brightness, because it is low in the infrared band and nonnegligible in the $R$ band. The value of this correction, $1.2 \mathrm{mag}$, is chosen so that the 2MASS sample coincides with our HSB objects in Figure 12.

Figure 12 shows all three samples together; the 2MASS objects are denoted by the triangles. A trend is seen in Figure 12: an average difference of $2 \mathrm{mag}$ in $\mu_{0}$ leads to a factor of 1.5 change in the ratio of scales. At the same time, there is no clear correlation found when $h$ and $z_{0}$ are plotted against $\mu_{0}$ separately. The correlation of $\mu_{0}$ versus $h$ was shown by Graham (2001), but that conclusion was based on mostly early-type spiral galaxies.

We also incorporate general galactic properties taken from the LEDA database into the analysis: absolute $B$ magnitude $B_{\text {abs }}$, maximum of the rotation curve $V_{\max }$, and $\mathrm{H}$ I index. The latter index denotes the difference between the $B$ magnitude and the $\mathrm{H}$ I magnitude. We found that LSB and HSB subsamples do not differ systematically in $B_{\mathrm{abs}}, V_{\max }$, and $\mathrm{H}_{\mathrm{I}}$ index. There is no

FIG. 6.-Same as Fig. 1, but for UGC 7808. The isophotes correspond to $20.5,21,21.5,22,22.5,23,23.5,24,24.5$, and $25.5 \mathrm{mag} \operatorname{arcsec}^{-2}$.

correlation found between the values of $\mu_{0}$ and $z_{0} / h$ on the one hand and $B_{\mathrm{abs}}, V_{\max }$, and $\mathrm{H}$ I index on the other hand.

In Figure 13 one can see a relation of the Tully-Fisher type, where the values of the radial scale length are well correlated with the maximum rotational velocity $V_{\max }$. According to Zwaan et al. (1995), Sprayberry et al. (1995), and Chung et al. (2002), LSB and HSB spiral galaxies follow the same TullyFisher relation, and our Figure 13 is in good agreement with this, which argues that we did not make a mistake deriving the spatial values. Thus, in their investigation of the galaxy UGC 7808, de Grijs \& van der Kruit (1996) inferred a shorter scale height value (in $\mathrm{kpc}$ ) because of the lower adopted distance to the galaxy. Figure 13 shows that our value of the scale length for the galaxy, $13.55 \mathrm{kpc}$, places the galaxy very close to the general dependence in Figure 13, whereas the scale length of 1.9-2.7 kpc taken from de Grijs \& van der Kruit (1996) would place this object far off. At the same time, the angular values of the scale lengths found in the present work and in the cited one are very similar.

Following Zasov et al. (2002), we calculated the ratio of the total mass $M_{t}$ inside the optical radius to the luminosity of the galactic disk in the $B$ band $L_{B}$. We suppose that $M_{t}=$ $G^{-1} 4 h V_{\max }^{2}$, where $G$ is the gravitational constant and the $4 h$ radius encompasses the whole galaxy. The value of $L_{B}$ is obtained from the absolute $B$ magnitude, which was taken from the LEDA and corrected for the internal galactic absorption. The values of $M_{t} / L_{B}$ are plotted against the ratio $z_{0} / h$ in Figure 14. 

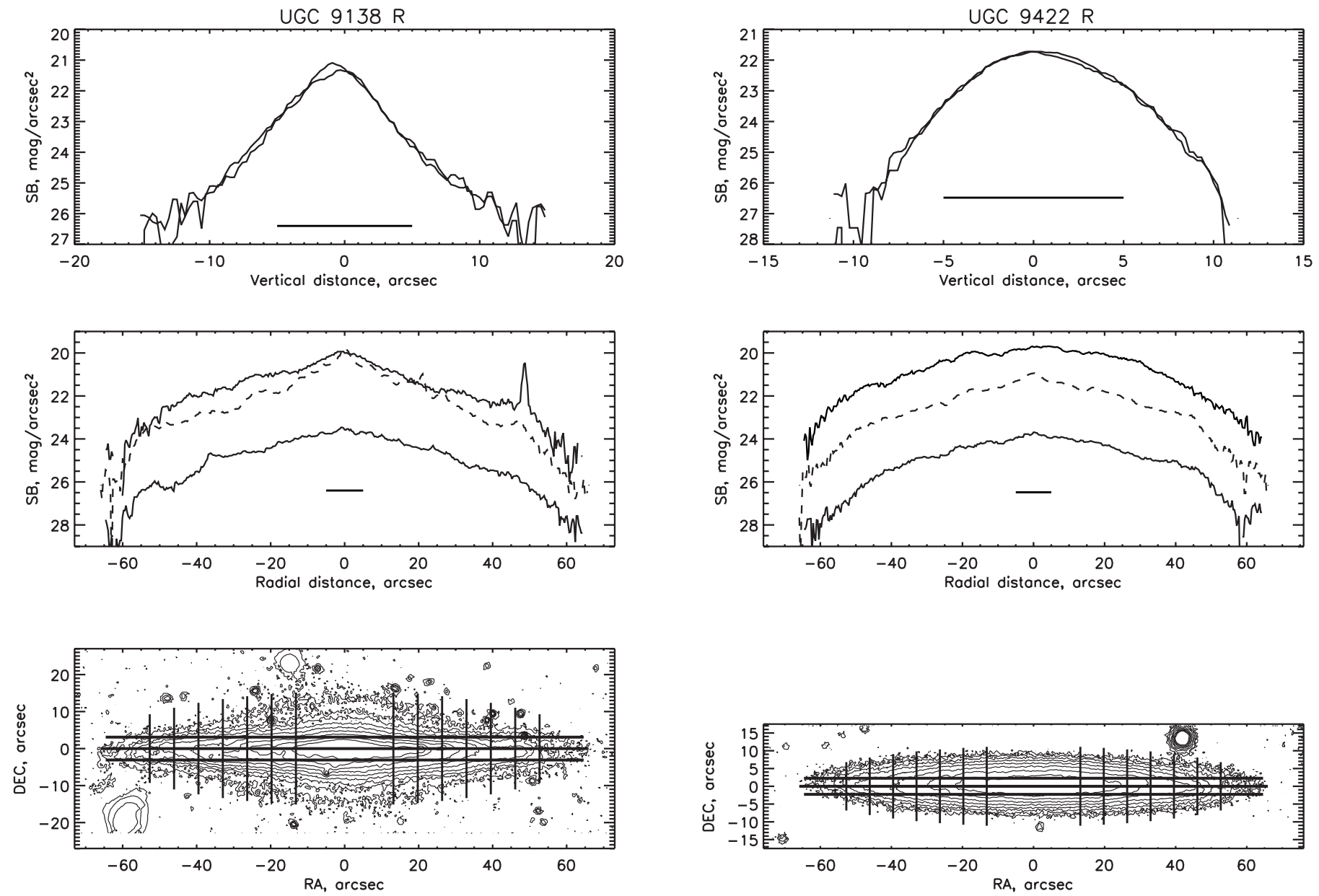

FIG. 7.-Same as Fig. 1, but for UGC 9138. The isophotes correspond to $20.5,21,21.5,22,22.5,23,23.5,24,24.5,25$, and $26 \mathrm{mag} \mathrm{arcsec}^{-2}$.

The notation in Figure 14 is the same as that in Figure 12. As noticed by Zasov et al. (1991), the ratio of scales $z_{0} / h$ indicates the total mass of the spherical component of a galaxy expressed in its disk mass $M_{s} / M_{d}$. The relation between $z_{0} / h$ and $M_{s} / M_{d}$ obtained from numerical modeling ( $N$-body simulations) was published by Mikhailova et al. (2001) and is shown in Figure 15. We made use of that dependence to evaluate the model values of $M_{s} / M_{d}$ for our galaxies.

Here we must clarify that we distinguish between spherical and disk subsystems throughout this paper. By the term "spherical subsystem" we refer to both a stellar bulge and a dark halo, even if their shapes are not spherical but oblate (see discussion in $\S 5.5$ ). In general, "spherical subsystem" means a nondisk component, whether stellar or not. The disk in our understanding is the galactic stellar disk, which for our objects consists mostly of stars. Later in this paper we also evaluate the ratio of dark-to-luminous masses. The dark mass refers to the dark halo, whereas the luminous mass refers to the stellar bulge and disk.

Then we take into account that $M_{t}=M_{s}+M_{d}$ and $L_{B}=$ $M_{d} /(M / L)$, where $M / L$ denotes the $B$-band stellar mass-to-light ratio in the disk. Hence $M_{t} / L_{B}=\left(M_{s} / M_{d}+1\right)(M / L)$. We see that the model value of $M_{t} / L_{B}$ depends on the adopted $M / L$. The three lines in Figure 14 present the model values of $M_{t} / L_{B}$, which were calculated based on Figure 15 with $M / L$ values of 1, 5, and 15, respectively. As shown in Figure 14, most of the galaxies have $M / L$ values between 3 and 10 . The $M / L$ in

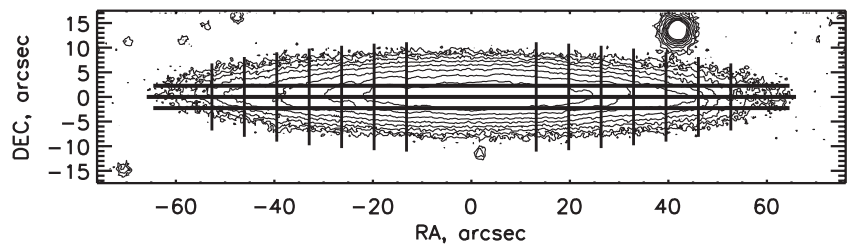

FIG. 8.-Same as Fig. 1, but for UGC 9422. The isophotes correspond to $20.5,21,21.5,22,22.5,23,23.5,24,24.5,25$, and 25.5 mag $\operatorname{arcsec}^{-2}$.

Figure 14 corresponds to the distance along the horizontal axis toward the curve of $M / L=1$. The value of the stellar mass-tolight ratio is systematically higher for our LSB galaxies than for HSB galaxies.

This conclusion contradicts the bluer color of LSB galaxies found by de Blok et al. (1995), who give lower values for their $M / L$, but the bulge-dominated LSB galaxies have colors comparable with HSB galaxies (Beijersbergen et al. 1999). The dereddened colors from both our LSB and HSB subsamples are almost the same (Table 3). On the other hand, LSB spirals have low metallicity, as a rule. This might give the comparable colors, whereas the stellar disk's $M / L$ has a higher value in the LSB spirals. Another reasonable explanation might be an excess of dark matter in the disks of bulge-dominated LSB spirals.

Large LSB galaxies have, on average, twice the mass in their gaseous components (Romanishin et al. 1982) as HSB galaxies. Our LSB subsample has almost twice the $\mathrm{H}$ I index of the HSB subsample. However, this difference is not enough to explain the systematic difference in $M / L$ in Figure 14 , since the gas component does not dominate by mass in our galaxies.

The mass of the dark halo $M_{h}$ can be estimated from the relation shown in Figure 15. The dark-to-luminous ratio is $\left(M_{d}+M_{b}\right) / M_{h}=\left(1+M_{b} / M_{d}\right)\left(M_{d} / M_{h}\right)$, where $M_{b}$ and $M_{d}$ denote the masses of the bulge and the dark halo, respectively. On the other hand, $M_{s} / M_{d}=\left(M_{h}+M_{b}\right) / M_{d}$, hence, 

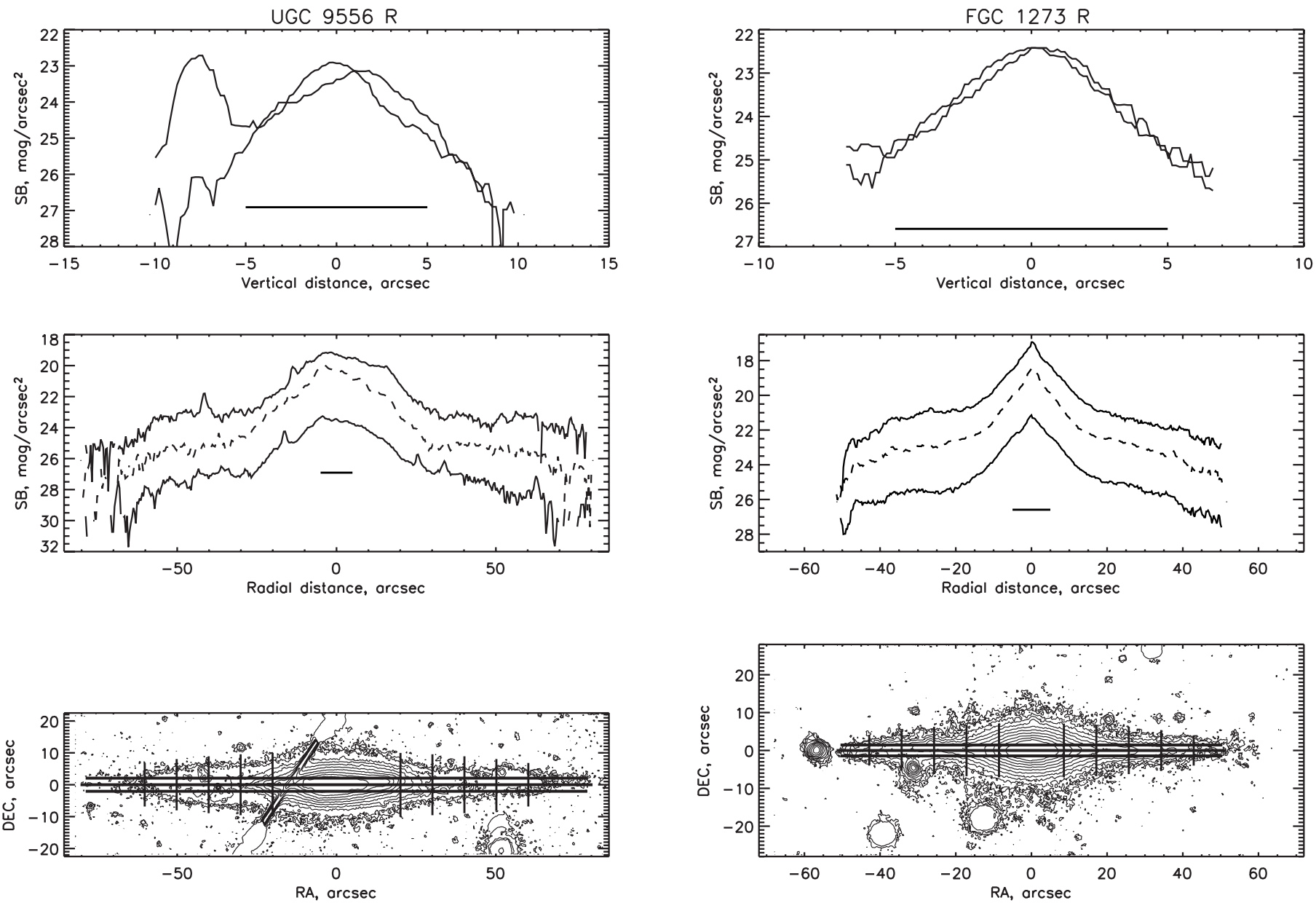

FIG. 9.- Same as Fig. 1, but for UGC 9556. The isophotes correspond to $20.5,21,21.5,22,22.5,23,23.5,24,24.5,25,26$, and $26.7 \mathrm{mag} \mathrm{arcsec}^{-2}$. The diagonal feature in the bottom panel is a remainder of a bright satellite track.

$M_{h} / M_{d}=M_{s} / M_{d}-M_{b} / M_{d}$. Combining previous equations, we obtain

$$
\frac{M_{h}}{M_{d}+M_{b}}=\frac{M_{s} / M_{d}-M_{b} / M_{d}}{1+M_{b} / M_{d}} .
$$

The values of $M_{b} / M_{d}$ can be estimated from observations making a rough assumption that the bulge-to-disk luminosity ratio follows the bulge-to-disk mass ratio, $M_{b} / M_{d}=L_{b} / L_{d}$ (we consider in $\S 5$ how our conclusions might change for real galaxies where $M / L$ is different for bulges and disks). At the same time, $M_{s} / M_{d}$ can be estimated from Figure 15 . The ratio of dark-to-luminous mass $M_{h} /\left(M_{d}+M_{b}\right)$ for our galaxies is shown in Figure 16. Surprisingly, there is no systematic difference between the values of dark-to-luminous mass ratio for galaxies with different central surface brightnesses (see Fig. 16). It is generally assumed that the LSB galaxies are dark matter dominated, but those conclusions were based on studies of bulgeless galaxies. Our sample, however, comprises mostly galaxies possessing nonnegligible bulges.

We can also compare masses of the spherical subsystem $M_{s}$ (i.e., the sum of the bulge and the halo) in our galaxies. In Figure 17 we present how the spherical-to-disk mass ratio $M_{s} / M_{d}$ depends on the disk central surface brightness. We used the same notation as in Figures 12 and 14. Figure 17 indicates that the LSB galaxies do not have more massive dark matter halos. Instead, they have more massive spherical subsystems.

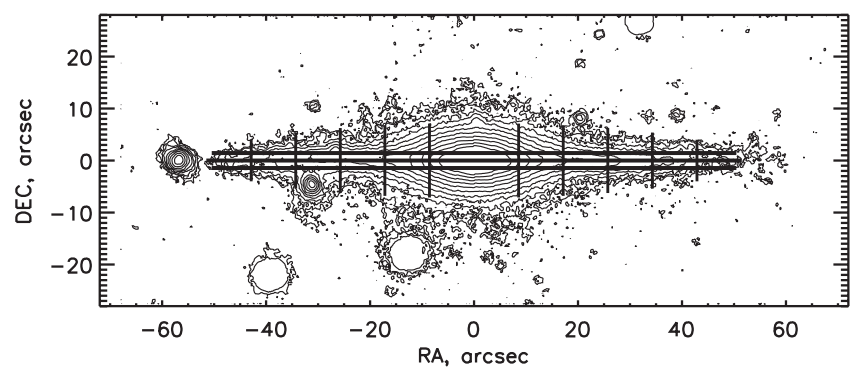

FIG. 10.-Same as Fig. 1, but for FGC 1273. The isophotes correspond to $20.5,21,21.5,22,22.5,23,23.5,24,24.5,25$, and 25.5 mag $\operatorname{arcsec}^{-2}$.

This supports a result by Graham (2002) that not all LSB galaxies are dark matter dominated objects. Nevertheless, our result does not contradict previous conclusions, since the dark matter halo and the spherical subsystem become identical for bulgeless galaxies.

Differentiation between the bulge and the halo allows us to demonstrate that there are large LSB galaxies dominated by dark matter halos as well as LSB galaxies with halos less massive than their disks.

\section{DISCUSSION}

\subsection{The Sample Selection}

There is no systematic difference in the obtained values of $\mu_{0}$ among our sample galaxies of I-III SB class (also noticed by Bizyaev 2000). On the other hand, most galaxies of IV SB class are apparently bona fide LSB galaxies. They constitute a small part of all RFGC objects (3\%). As noticed by McGaugh et al. (1995), there is a significant fraction of LSB galaxies with a large bulge-to-disk ratio. Bulges of LSB and HSB systems are indistinguishable (Beijersbergen et al. 1999), yet their disks are different. Hence, we must distinguish between LSB galaxies with bulges and without bulges and take this into account when studying properties of dark halos in LSB galaxies.

Our paper does not attempt to present a statistically complete study of LSB spiral galaxies with large bulges. Instead, we compare two samples of objects of opposite properties. To 

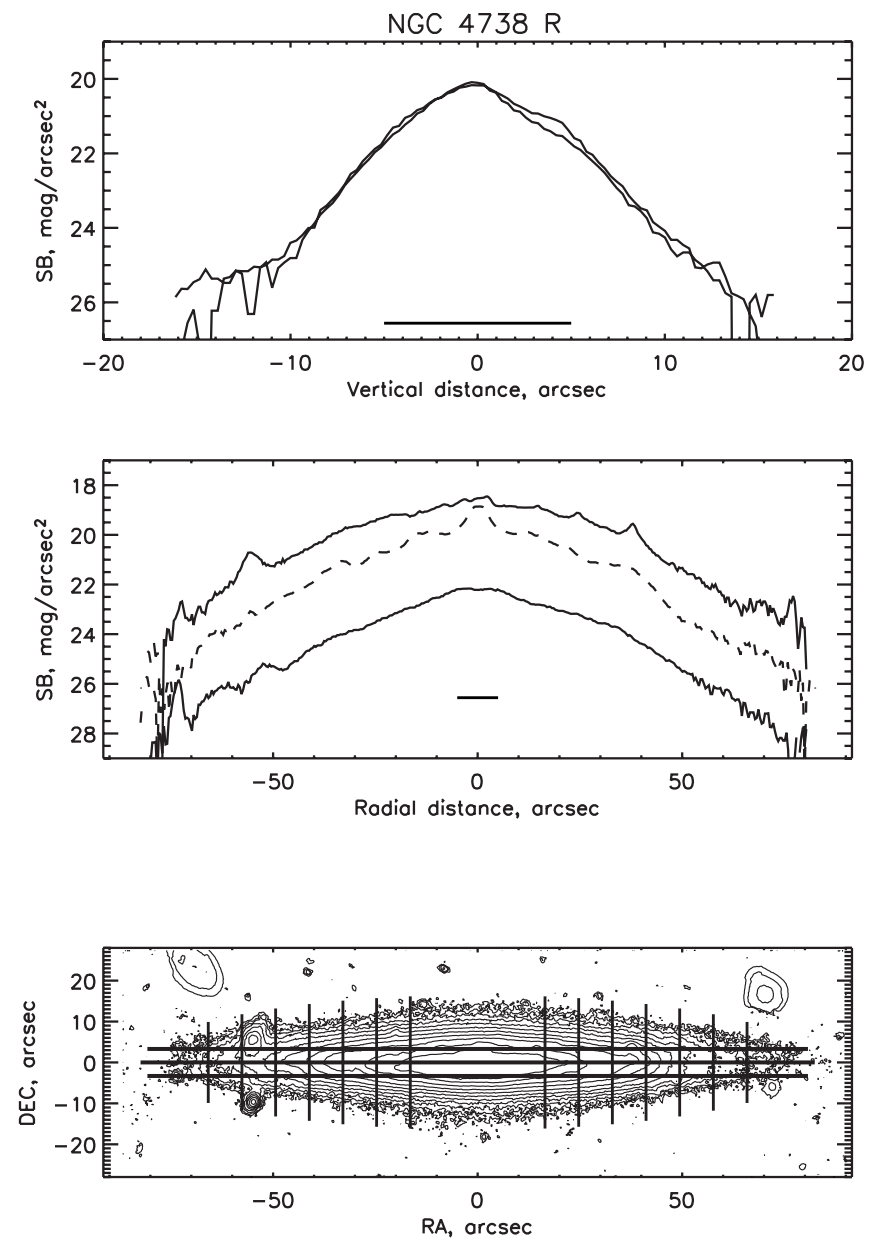

FIG. 11.-Same as Fig. 1, but for NGC 4738. The isophotes correspond to $20.5,21,21.5,22,22.5,23,23.5,24,24.5,25$, and $25.5 \mathrm{mag} \mathrm{arcsec}^{-2}$.

make statistically reliable conclusions, the sample has to be extended.

\subsection{Selection Effects}

Figure 12 presents a correlation between $\mu_{0}$ and $z_{0} / h$. Indeed, the values of $\mu_{0}$ and $z_{0} / h$ have not been obtained independently from each other, as can be concluded from the formulae in $\S 3$. Let us consider how the non- $90^{\circ}$ inclination of the disk plane affects $\mu_{0}$ and $z_{0} / h$. If the inclination angle is less than $90^{\circ}$, the scale height $z_{0}$ calculated in $\S 3$ will be overestimated. At the same time, the scale length $h$ is much less affected by the value of the inclination angle. On the other hand, the value of $z_{0}$ was taken into account when the central surface brightness was calculated. While overestimating the ratio $z_{0} / h$, we underestimate the disk central surface brightness (hence, its numerical value will be larger). This means that a non- $-90^{\circ}$ inclination of the disk shifts the data points in Figure 12 toward the upper right corner. Hence, the systematic errors due to inclination may only scatter the dependence shown in Figure 12 (for example, for the 2MASS galaxies) and do not explain a good correlation.

The second effect that has to be considered is the internal dust absorption in galaxies. According to Xilouris et al. (1999), the scale length in dusty disks appears higher because of the scattering and absorption effects. On the other hand, the dust absorption decreases the derived central surface brightness. In our case it would shift the data points in Figure 12 from the upper left corner to the lower right corner and form a dependence similar to that seen in Figure 12. Nevertheless, we decreased the influence of dust by avoiding the dust layer when extracting the radial profiles. This allowed us to minimize the dust absorption. Furthermore, one can see that the infrared and optical subsamples follow a dependence similar to that seen in Figure 12. This argues that the internal absorption has little effect on the difference between LSB and HSB photometric parameters and that Figure 12 has a physical meaning.

\subsection{Internal Absorption in Galactic Disks and the Ratios $M_{s} / M_{d}$ and $M_{h} /\left(M_{b}+M_{d}\right)$}

As noted in $\S 4$, the disk thickness is different when it is estimated in different photometric bands. All the considered relations between the mass of a dark halo, stellar disk, and spherical component are made using the data taken in the $R$ band. On the other hand, the infrared ratios of photometric scales $z_{0} / h$ are less than the optical ratios. The infrared values are preferable because of the lower dust absorption, so we could decrease all our ratios $z_{0} / h$ by a factor of 1.4. As shown in Figures 14 and 15, a proportional decrease of the scale ratio affects Figures 16 and 17 only quantitatively. Hence, all previous conclusions remain unchanged.

TABLE 2

General Galactic Parameters Used in the Paper

\begin{tabular}{|c|c|c|c|c|c|c|c|}
\hline Name & $\begin{array}{c}D \\
(\mathrm{Mpc})\end{array}$ & Type & $\log D_{25}$ & $B_{t}$ & $A_{B}$ & $\log V_{\max }$ & $B_{\text {abs }}$ \\
\hline UGC $10111 \ldots \ldots \ldots$ & 139.6 & $\mathrm{Sc}$ & 1.221 & 16.0 & 0.178 & 2.370 & -21.3 \\
\hline UGC $11301 \ldots \ldots \ldots . .$. & 62.3 & $\mathrm{Sc}$ & 1.295 & 15.5 & 1.273 & 2.379 & -21.2 \\
\hline UGC $5662 \ldots \ldots \ldots \ldots . . . .$. & 17.1 & $\mathrm{SBb}$ & 1.495 & 15.4 & 0.115 & 1.899 & -17.6 \\
\hline UGC $6080 \ldots \ldots \ldots . . .$. & 30.3 & Scd & 1.3 & 15.8 & 0.036 & 1.877 & -18.6 \\
\hline UGC $6686 \ldots \ldots \ldots \ldots$ & 86.4 & $\mathrm{Sb}$ & 1.418 & 15.0 & 0.135 & 2.283 & -21.2 \\
\hline UGC $7808 \ldots \ldots \ldots \ldots . . . .$. & 96.3 & $\mathrm{Sb}$ & 1.492 & 14.6 & 0.098 & 2.403 & -21.8 \\
\hline UGC $9138 \ldots \ldots \ldots \ldots$ & 61.9 & $\mathrm{Sc}$ & 1.284 & 14.8 & 0.108 & 2.161 & -20.9 \\
\hline UGC $9422 \ldots \ldots \ldots \ldots . . . .$. & 45.6 & $\mathrm{Sc}$ & 1.279 & 14.7 & 0.1 & 2.140 & -20.5 \\
\hline UGC $9556 \ldots \ldots \ldots \ldots . . . .$. & 32.5 & $\mathrm{SBc}$ & 1.099 & 16.0 & 0.043 & 1.974 & -18.1 \\
\hline FGC $1273 \ldots \ldots \ldots \ldots$ & 49.4 & $\mathrm{Sc}$ & 0.801 & 16.5 & 0.103 & 2.166 & -18.0 \\
\hline NGC $4738 \ldots \ldots \ldots \ldots$ & 63.6 & $\mathrm{Sc}$ & 1.297 & 14.3 & 0.076 & 2.335 & -21.4 \\
\hline
\end{tabular}

Note.-Name of galaxy, adopted distance (corresponding to the Hubble constant $H_{0}=75 \mathrm{~km} \mathrm{~s}^{-1} \mathrm{Mpc}^{-1}$ ), morphological type, major axis size $\log D_{25}$ (in 0.1 ), total $B$-band magnitude, foreground extinction in our Galaxy in $B$, logarithm of rotational velocity $\log V_{\max }$, and absolute $B$ magnitude (all values taken from the LEDA). 
TABLE 3

The Derived Structural Parameters of the Galaxies

\begin{tabular}{|c|c|c|c|c|c|c|c|c|c|}
\hline Name & $\begin{array}{c}i \\
(\operatorname{deg})\end{array}$ & $\begin{array}{l}\text { P.A. } \\
\text { (deg) }\end{array}$ & $\begin{array}{c}h \\
(\mathrm{kpc})\end{array}$ & $\begin{array}{c}z_{0} \\
(\mathrm{kpc})\end{array}$ & $z_{0} / h$ & $\begin{array}{c}\mu_{0} \\
\left(\mathrm{mag}_{\operatorname{arcsec}}^{-2}\right)\end{array}$ & $L_{b} / L_{d}$ & $\begin{array}{c}m_{R} \\
(\mathrm{mag})\end{array}$ & $\begin{array}{l}V-R \\
(\mathrm{mag})\end{array}$ \\
\hline UGC $10111 \ldots \ldots \ldots \ldots$ & 88.2 & 37.5 & $15.84 \pm 0.14$ & $2.60 \pm 0.42$ & 0.168 & $24.63 \pm 0.11$ & 0.58 & 15.08 & 0.63 \\
\hline UGC $11301 \ldots \ldots \ldots \ldots$ & 88.2 & 110.0 & $8.24 \pm 0.84$ & $1.30 \pm 0.08$ & 0.160 & $22.12 \pm 0.13$ & 0.25 & 13.01 & 0.81 \\
\hline UGC $5662 \ldots \ldots \ldots \ldots . . .$. & 89.3 & 147.5 & $2.16 \pm 0.51$ & $0.50 \pm 0.06$ & 0.237 & $22.70 \pm 0.37$ & 0.21 & 13.62 & 0.61 \\
\hline UGC $6080 \ldots \ldots \ldots \ldots . . . .$. & $86(?)$ & 125.0 & $2.93 \pm 0.18$ & $0.70 \pm 0.05$ & 0.236 & $22.69 \pm 0.16$ & 0.00 & 14.45 & 0.50 \\
\hline UGC $6686 \ldots \ldots \ldots \ldots . . . .$. & 88.0 & 50.0 & $9.82 \pm 1.27$ & $1.89 \pm 0.21$ & 0.196 & $22.51 \pm 0.13$ & 0.10 & 13.44 & 0.71 \\
\hline UGC $7808 \ldots \ldots \ldots \ldots . . . .$. & 88.7 & 93.5 & $13.55 \pm 2.63$ & $1.44 \pm 0.18$ & 0.158 & $23.97 \pm 0.18$ & 0.86 & 13.60 & 0.69 \\
\hline UGC $9138 \ldots \ldots \ldots \ldots . . . .$. & 87.0 & 168.5 & $4.71 \pm 0.12$ & $1.05 \pm 0.06$ & 0.214 & $22.42 \pm 0.38$ & 0.07 & 14.79 & 0.76 \\
\hline UGC $9422 \ldots \ldots \ldots \ldots . . . .$. & 88.3 & 159.0 & $3.54 \pm 0.13$ & $0.80 \pm 0.05$ & 0.225 & $22.64 \pm 0.35$ & 0.00 & 14.96 & $\ldots$ \\
\hline UGC $9556 \ldots \ldots \ldots \ldots . . . .$. & 87.0 & 133.0 & $3.63 \pm 0.49$ & $0.54 \pm 0.09$ & 0.141 & $24.77 \pm 0.60$ & 1.00 & 15.39 & 0.39 \\
\hline FGC $1273 \ldots \ldots \ldots \ldots . . .$. & 89.7 & 170.0 & $5.17 \pm 0.98$ & $0.63 \pm 0.10$ & 0.118 & $24.33 \pm 0.18$ & 1.37 & 14.70 & 0.68 \\
\hline NGC $4738 \ldots \ldots \ldots \ldots . . . .$. & 85.7 & 32.5 & $4.68 \pm 0.21$ & $1.23 \pm 0.08$ & 0.270 & $21.11 \pm 0.16$ & 0.01 & 13.39 & $\ldots$ \\
\hline
\end{tabular}

NoтE.-Parameters derived for our galaxies: inclination angle, position angle, disk scale length in kiloparsecs, disk scale height, ratio of scales $z_{0} / h$, stellar disk central surface brightness, bulge-to-disk ratio, $R$ magnitude integrated within the elliptical diaphragm with axis size taken from the RFGC catalog, and color $(V-R)$. The $R$ magnitude and the color are uncorrected for the foreground extinction.

The values of $\mu_{0}$ inferred for our HSB galaxies are less than Freeman's value (taking into account a difference between the $R$ and $B$ bands). This implies that the internal extinction may be important in the disks of our galaxies. Since all the galaxies are spiral and are relatively nearby, one can assume they have roughly the same ratio of dust to stars. Thus, the internal extinction proportionally increases the values of $\mu_{0}$. At the same time, it does not change the main trends in Figures 12, 14, 16, and 17.

In a more complicated case, the internal dust extinction may be systematically different in the galaxies of our sample. Thus, according to McGaugh (1994) and Matthews \& Wood (2001), LSB spirals are likely to be less dusty than HSB spirals. One can see that this strengthens the relation shown in Figure 12:

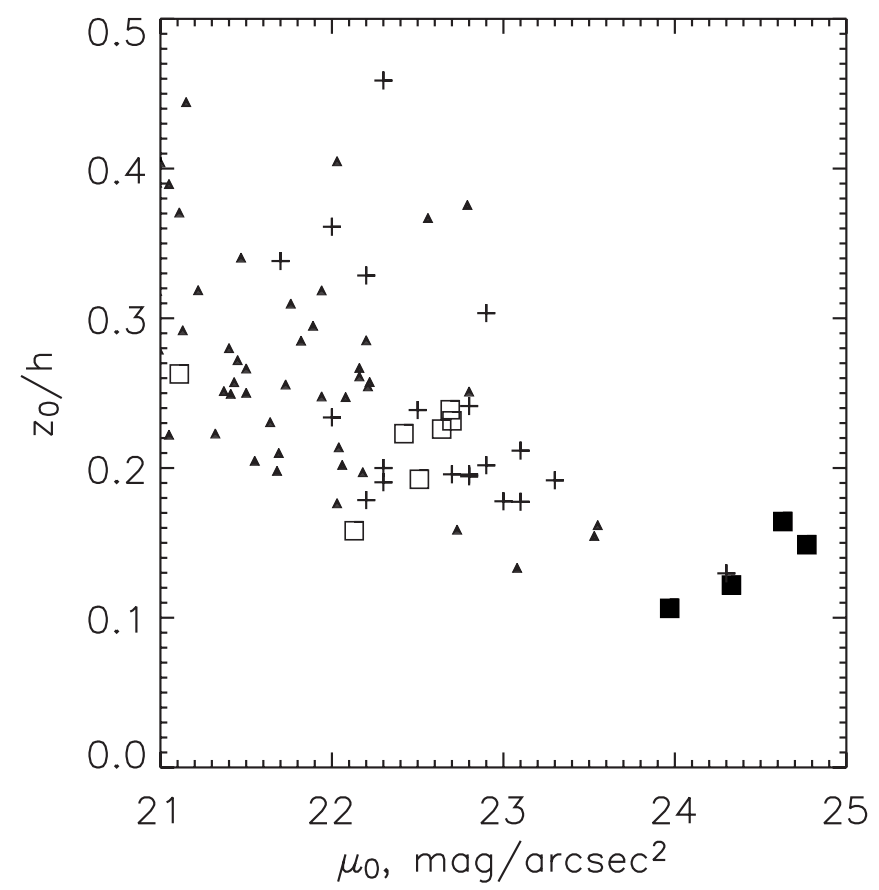

FIG. 12.-Vertical-to-radial scales ratio of the stellar disks $z_{0} / h$ vs. their central surface brightness $\mu_{0}$ in the $R$ band. The objects from our sample are denoted by squares. The open squares are for the HSB subsample, and the filled ones designate the LSB galaxies. The galaxies taken from Barteldrees \& Dettmar (1994) are shown as crosses, and the 2MASS objects are denoted by triangles. extinction correction of $\mu_{0}$ for HSB spirals moves the data points further to the left than it does for LSB spirals. As a result, we can always distinguish between these two subsamples. This corresponds to the conclusion by Beijersbergen et al. (1999) that the dust extinction alone cannot explain the difference in surface brightness between LSB and HSB spirals.

Another way to take the extinction into account is to connect it to global galactic parameters such as the absolute magnitude or rotational velocity (see Tully et al. 1998 and references therein). Correction of $\mu_{0}$ for the extinction with the help of absolute $R$ magnitudes or $V_{\max }$ moves the data points to the left in Figure 12 but does not change the figure's general trend.

The bulge-to-disk luminosity ratio has been used to draw Figures 12 and 16. Since some of our galaxies have bulges, attention should be paid to how the extinction may change the

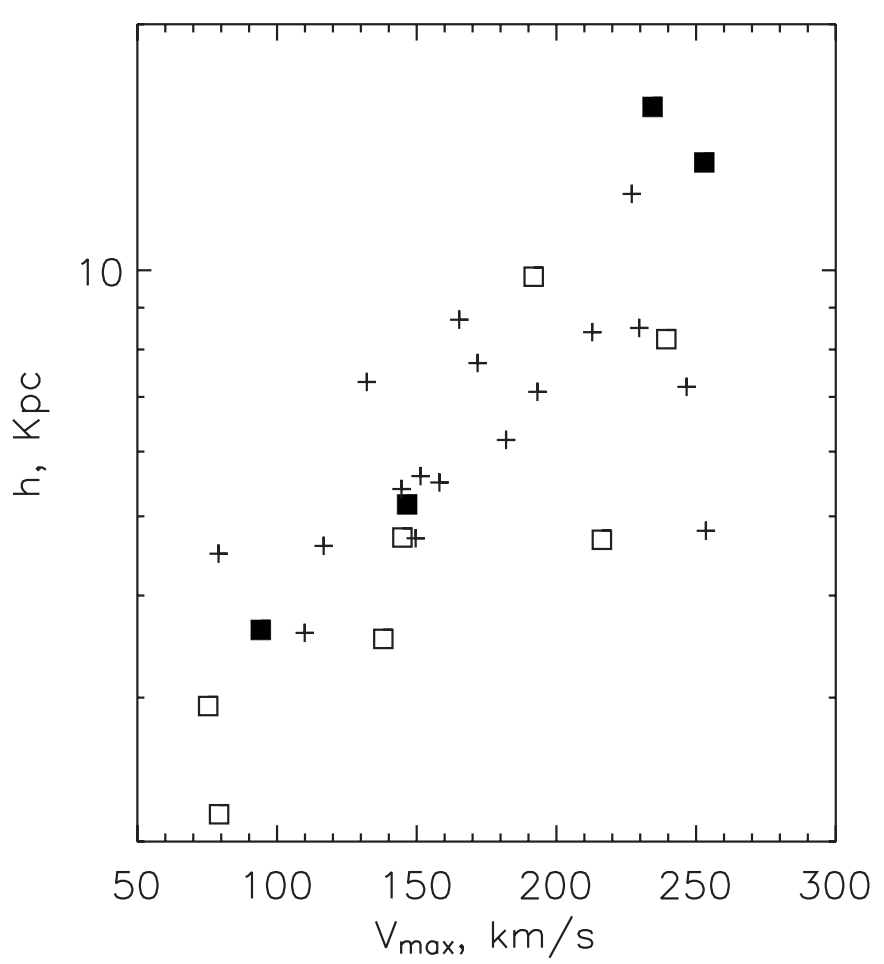

FIG. 13.-Radial scale length $h$ well correlated with the maximum rotational velocity $V_{\max }$. The notation is the same as in Fig. 12 . 


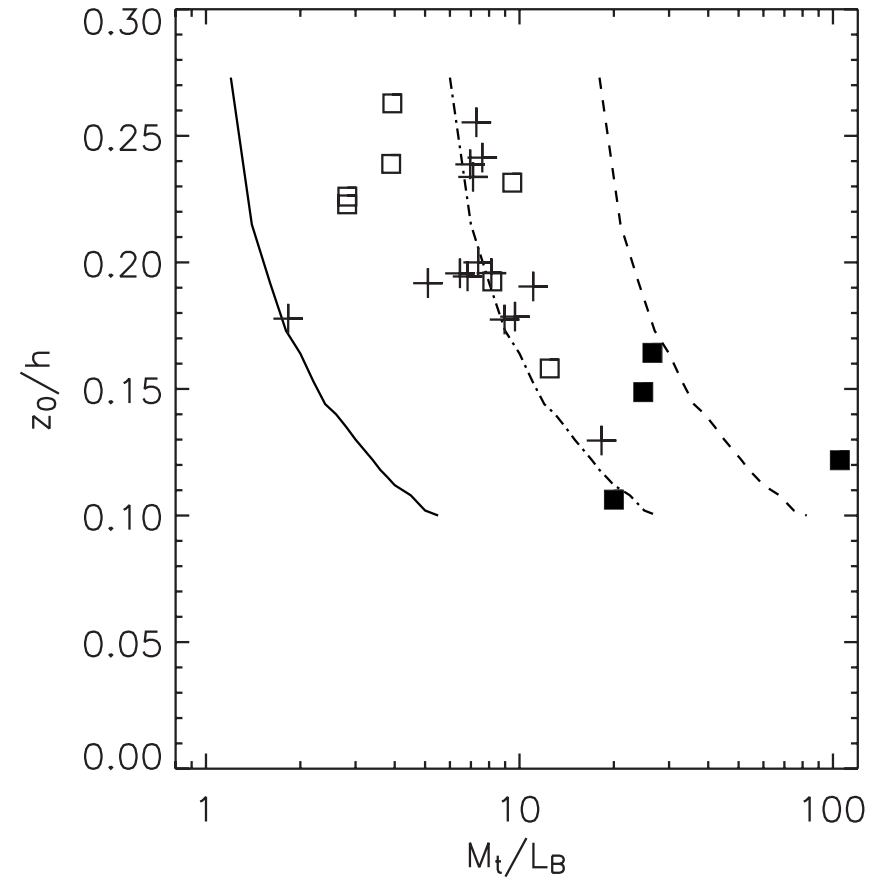

Fig. 14.- Ratio of total mass to $B$-luminosity of disk $M_{t} / L_{B}$ (see text for details) plotted against the disk scales ratio $z_{0} / h$. The notation in the figure is the same as in Fig. 12. The three lines represent the model values of $M_{t} / L_{B}$, which were calculated based on Fig. 15 with mass-to-light ratios of 1 (solid line), 5 (dot-dashed line), and 15 (dashed line).

derived values of $L_{b} / L_{d}$. In addition to the profile fitting, we conducted a direct integration of bulges. At first, the model disk (constructed according to the parameters defined during the disk fitting) was subtracted from the images of galaxies. Then we integrated a portion of the central part that was above

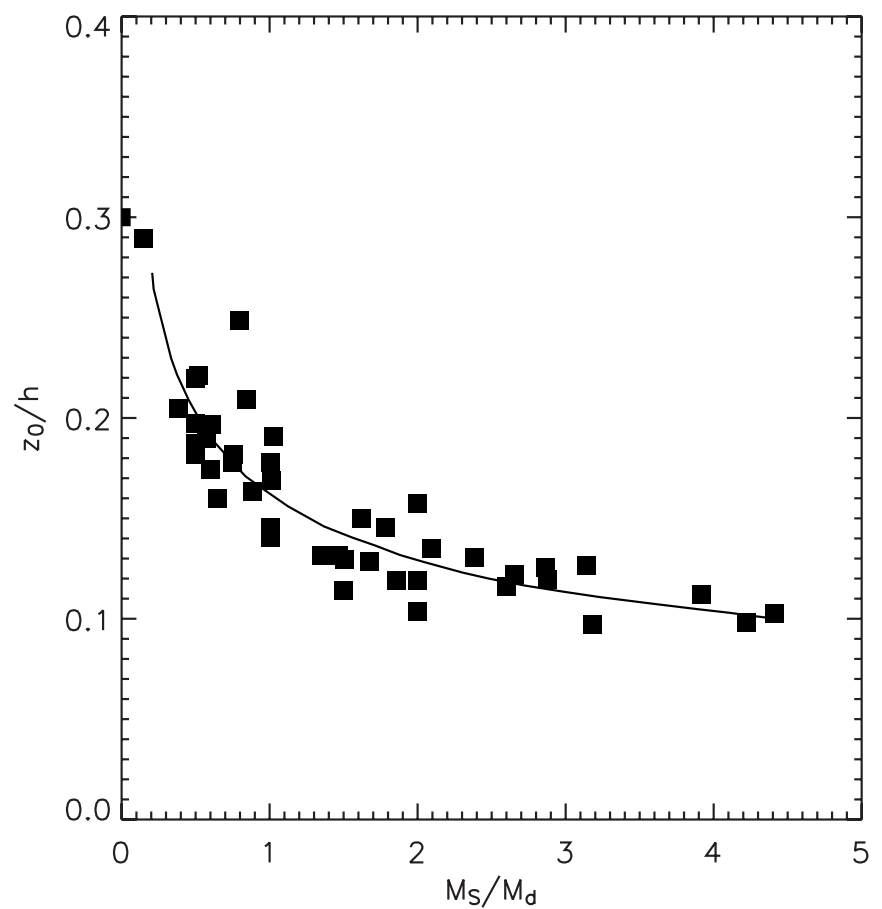

Fig. 15.-Relation between the stellar disk thickness $z_{0} / h$ and the relative mass of its spherical component $M_{s} / M_{d}$ obtained from numerical simulations ( $N$-body experiments). The figure is adopted from Mikhailova et al. (2001); see that paper for details.

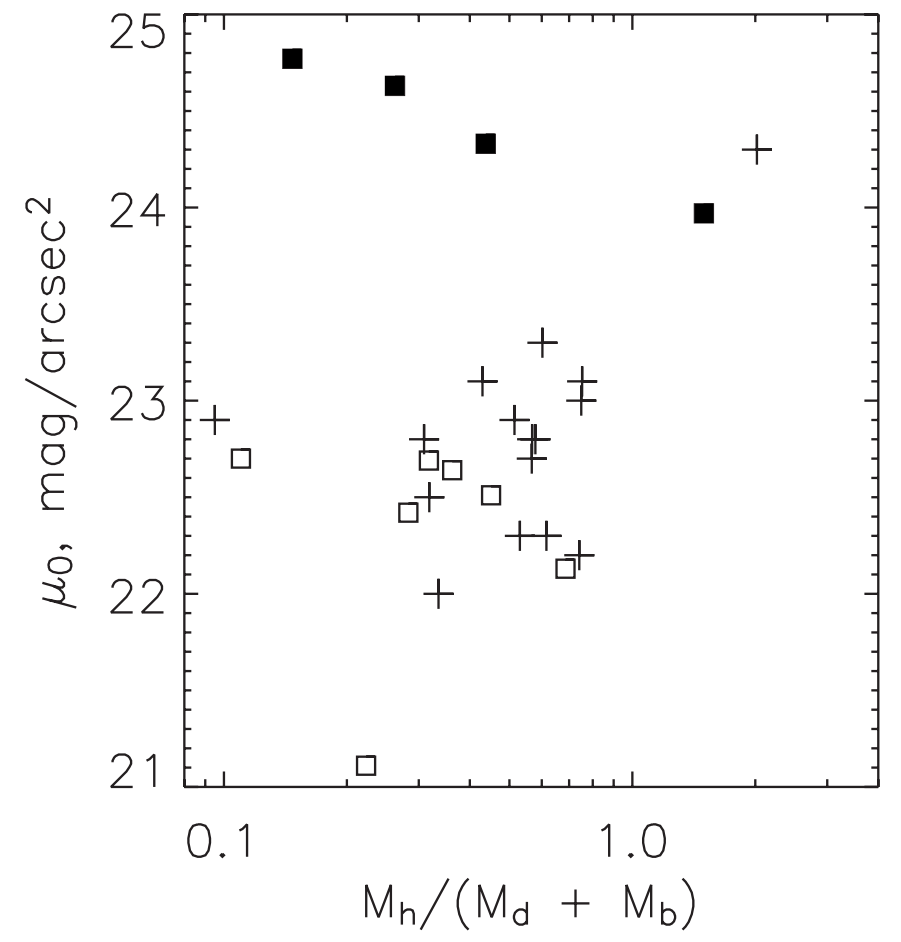

FIG. 16.- Ratio of dark-to-luminous mass $M_{h} /\left(M_{d}+M_{b}\right)$ for our galaxies. The notation in the figure is the same as in Fig. 12.

the zero level. The ratio of the integrated luminosity of the bulge to the model disk luminosity $L_{b}^{I} / L_{d}$ gives us a lower bound of $L_{b} / L_{d}$ (because the model disk is "dust-free" and the bulge is dimmed by the extinction). The value of $L_{b}^{I} / L_{d}$ is 2-6 times lower than the value of $L_{b} / L_{d}$ given in Table 3 . If we use $L_{b}^{I} / L_{d}$ instead of $L_{b} / L_{d}$, Figure 12 does not change qualitatively. However, in Figure 16, all of our LSB galaxies

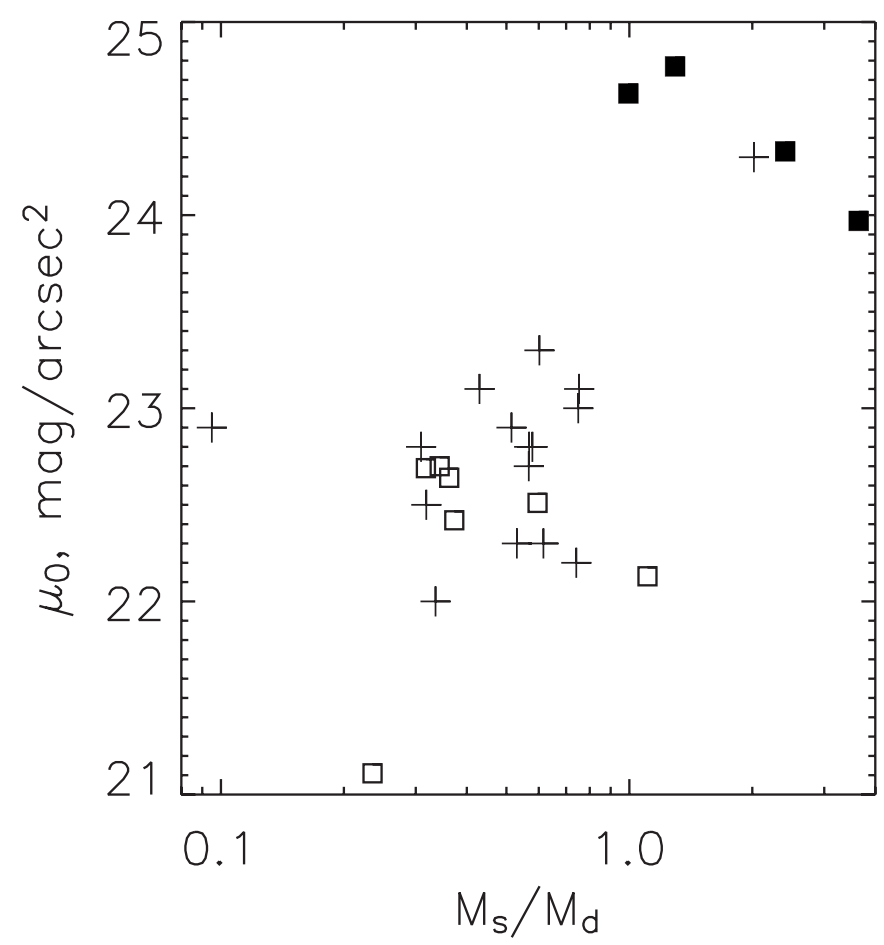

FIG. 17.-Dependence of the spherical-to-disk mass ratio $M_{s} / M_{d}$ for our galaxies on the disk central surface brightness. The notation in the figure is the same as in Fig. 12. 
move to the right, since a larger fraction of the mass of their spherical component is assigned to the dark halo. Then if we apply $L_{b}^{I} / L_{d}$ as a bulge-to-disk luminosity ratio, we cannot conclude that the ratio (dark halo + bulge)/disk in the bulgedominated galaxies is systematically higher while the dark-toluminous ratio is not. In this case the dark-to-luminous mass ratio would also be higher in our LSB systems.

Alternatively, we can obtain the ratio $L_{b} / L_{d}$ from direct integration of the bulge and disk from our images. In contrast to the previous case, which gives the lower limit on $L_{b} / L_{d}$, this integration yields values of $L_{b} / L_{d}$ that are systematically higher than those shown in Table 3 . This method of evaluation of bulgeto-disk luminosity ratio also does not change our conclusions.

\subsection{M/L May Be Different for Bulge and Disk}

Assuming that $M_{b} / M_{d}=L_{b} / L_{d}$, we notice that bulges and disks have different colors and, hence, their stellar populations must show different mass-to-light ratios. This does not affect our results, with the exception of equation (1) and Figure 16. Bulges are redder than disks, as a rule (Peletier \& Balcells 1996), and have larger $M / L$. Therefore, $M_{h} /\left(M_{d}+M_{b}\right)$ is overestimated for galaxies with significant bulges (LSB galaxies in our sample). This supports our conclusion that the dark halo does not dominate in LSB galaxies that have big bulges. It should be noticed that the difference between the colors of bulges and disks is very small (Peletier \& Balcells 1996; Gadotti \& dos Anjos 2001), which makes the effect mentioned above insignificant.

\subsection{Oblate Bulges, Nonspherical Halos}

Dark matter halos and bulges of galaxies may be oblate rather than exactly spherical. Our definition of $M_{t}=G^{-1} 4 h V_{\max }^{2}$ works well in the case of spherical symmetry. In a general case, $M_{t}=\eta G^{-1} 4 h V_{\max }^{2}$, where $\eta$ is a dimensionless parameter, the value of which is determined by the mass distribution, and $\eta<1$ in the case of galaxies. If the whole mass of a galaxy were enclosed in a thin exponential disk, the parameter $\eta$ taken at $4 h$ distance from the center would be approximately equal to 0.5 (Freeman 1970). All other reasonable geometric cases represent a mixture of disk and spherical components and give $\eta$ between 0.5 and 1 . In the case of a nonspherical dark halo, the difference between LSB and HSB galaxies in Figure 14 (and, hence, in $M / L$ for the stellar disk) would be even more prominent, because $M_{t}$ calculated using $\eta$ is systematically lower for diskdominated HSB spirals than for bulge-dominated LSB galaxies in our sample. Note that once a nondisk component is presented in all our galaxies, the difference in $\eta$ becomes significantly less than a factor of 2 . At the same time, it does not change other conclusions of this paper.

The possible existence of a nonspherical, oblate component was not taken into account by Mikhailova et al. (2001). If one takes it into account, the general trend shown in Figure 14 remains unchanged. However, a systematic difference between the ellipticity of dark halos in LSB and HSB galaxies can significantly affect Figure 14. For instance, an assumption of oblate halos in LSB spirals and spherical halos in HSB spirals decreases the difference between the $M / L$ in stellar disks mentioned above, since it shifts the data points to the left (although by less than a factor of 2). On the other hand, we show that the dark halos are not likely to be too massive, and hence do not dominate by mass in our bulge-dominated LSB spirals. Therefore, the role of their nonspherical shapes is insignificant. It is doubtful that the systematic difference between the ellipticity of dark halos in LSB and HSB galaxies can affect our conclusions. Furthermore, if a significant fraction of dark matter in the bulge-dominated LSB galaxies is located in their disks, it helps to raise their $M / L$, as shown in Figure 14 . Note that one candidate for the dark matter, cold molecular clouds, could form a disklike subsystem (Pfenniger et al. 1994).

\section{CONCLUSION}

1. We present results of photometric observations of a sample of edge-on galaxies. Our sample includes four LSB and seven HSB galaxies. The photometric disk scales (both vertical and radial), disk central surface brightness, and bulge-to-disk luminosity ratios were derived.

2. Stellar disks of LSB galaxies are thinner (when parameterized by the ratio $z_{0} / h$ ) than those of HSB galaxies. There is a clear correlation between their central surface brightness and the vertical-to-radial scale ratios.

3. Although they have different central surface brightness and bulge-to-disk ratios, the LSB and HSB galaxies in our sample follow the same dependence of disk scale length versus the maximum rotational velocity.

4. Our LSB galaxies tend to harbor massive spherical subsystems (bulge + halo) and have higher mass-to-luminosity ratios in their disks than the HSB objects. Nevertheless, the dark halo is not strictly the most massive subsystem in our bulge-dominated LSB galaxies. The LSB spirals appear to be spherical subsystem dominated galaxies but not always dark matter dominated.

D. B. is supported by NASA/JPL through grant 99-04-OSS058. The project was partially supported by the Russian Foundation for Basic Research via grant 04-02-16518. We have made use of the LEDA database. We thank Verne Smith and Michael Endl for their comments on the manuscript and the anonymous referee whose commentaries and corrections essentially improved the paper. D. B. is grateful to A. Khoperskov and Eduard Vorobyov for fruitful discussions and help.
Barteldrees, A., \& Dettmar, R.-J. 1994, A\&AS, 103, 475

Beijersbergen, M., de Blok, W., \& van der Hulst, J. 1999, A\&A, 351, 903

Bizyaev, D. 2000, Soviet Astron. Lett., 26, 219

Bizyaev, D., \& Mitronova, S. 2002, A\&A, 389, 795

Chung, A., van Gorkom, J., O’Neil, K., \& Bothun, G. 2002, AJ, 123, 2387

de Blok, W. J. G., Bosma, A., \& McGaugh, S. 2003, MNRAS, 340, 657

de Blok, W. J. G., van der Hulst, J. M., \& Bothun, G. D. 1995, MNRAS,

274,235

de Grijs, R., Peletier, R., \& van der Kruit, P. 1997, A\&A, 327, 966

de Grijs, R., \& van der Kruit, P. 1996, A\&AS, 117, 19

de Jong, R. 1996, A\&A, 313, 377

Freeman, K. 1970, ApJ, 160, 811

\section{REFERENCES}

Gadotti, D., \& dos Anjos, S. 2001, AJ, 122, 1298

Graham, A. 2001, MNRAS, 326, 543

2002, MNRAS, 334, 721

Holley-Bockelmann, J. K., \& Mihos, J. C. 2001, BAAS, 33, 798

Karachentsev, I., Georgiev, T., Kajsin, S., Kopylov, A., Ryadchenko, V., \& Shergin, V. 1992, Astron. Astrophys. Trans., 2, 265

Karachentsev, I., Karachentseva, V., Kudrya, Y., Sharina, M., \& Parnovsky, S. 1999, Bull. Spec. Astrophys. Obs., 47, 5

Landolt, A. 1992, AJ, 104, 340

Matthews, L. D. 2000, AJ, 120, 1764

Matthews, L. D., \& Wood, K. 2001, ApJ, 548, 150

McGaugh, S. 1994, ApJ, 426, 135 
McGaugh, S., Rubin, V., \& de Blok, W. 2001, AJ, 122, 2381

McGaugh, S., Schombert, J., \& Bothun, G. 1995, AJ, 109, 2019

Mikhailova, E. A., Khoperskov, A. V., \& Sharpak, S. S. 2001, Proc. Int. Conf. on Stellar Dynamics: From Classic to Modern, ed. L. P. Ossipkov \& I. I. Nikiforov (St. Petersburg: Sobolev Astron. Inst.), 147

Nilson, P. 1973, Uppsala General Catalogue of Galaxies (Stockholm: Almqvist \& Wiksell)

Peletier, R., \& Balcells, M. 1996, AJ, 111, 2238

Pfenniger, D., Combes, F., \& Martinet, L. 1994, A\&A, 285, 79

Pohlen, M., Dettmar, R.-J., Lutticke, R., \& Aronica, G. 2002, A\&A, 392, 807

Reshetnikov, V., Dettmar, R.-J., \& Combes, F. 2003, A\&A, 399, 879

Romanishin, W., Krumm, N., Salpeter, E., Knapp, G., Strom, K. M., \& Strom, S. E. 1982, ApJ, 263, 94

Sprayberry, D., Bernstein, G., Impey, C., \& Bothun, G. 1995, ApJ, 438, 72
Tully, R. B., Pierce, M. J., Huang, J.-S., Saunders, W., Verheijen, M. A. W., \& Witchalls, P. L. 1998, AJ, 115, 2264

van der Kruit, P., Jimenez-Vicente, J., Kregel, M., \& Freeman, K. 2001, A\&A, 379,374

van der Kruit, P., \& Searle, L. 1981, A\&A, 95, 105

Xilouris, E. M., Alton, P. B., Davies, J. I., Kylafis, N. D., Papamastorakis, J., \& Trewhella, M. 1998, A\&A, 331, 894

Xilouris, E. M., Byun, Y., Kylafis, N., Paleologou, E., \& Papamastorakis, J. 1999, A\&A, 344, 868

Zasov, A., Bizyaev, D., Makarov, D., \& Tyurina, N. 2002, Soviet Astron. Lett., 28,527

Zasov, A., Makarov, D., \& Mikhailova, E. 1991, Soviet Astron. Lett., 17, 374 Zwaan, M., van der Hulst, J., de Blok, W., \& McGaugh, S. 1995, MNRAS, 273, L35 